\title{
MODELING AND CALCULATION OF AMPHIBIOUS ALL-TERRAIN VEHICLE EQUIPPED BY VIBRATION ENERGY HARVESTING DAMPERS
}

\author{
Vitaly Krupenin $^{1,2}$, George Nerubenko ${ }^{3}$, Pavel Kolomier ${ }^{4}$ \\ ${ }^{1}$ Mechanical Engineering Research Institute of the Russian Academy of Sciences, Russia; \\ ${ }^{2}$ Moscow Polytechnic University, Russia; ${ }^{3}$ NER*MAR Limited, Canada; \\ ${ }^{4} \mathrm{PK} \& \mathrm{MM}$ Engineering Ltd, Canada \\ krupeninster@gmail.com, optimalproject@hotmail.com,pk6662041@gmail.com
}

\begin{abstract}
The amphibious all-terrain vehicle chassis and its components are subjected to aggressive vibration fields during driving in agricultural regions. The system of the newly invented Vibration Energy Harvesting Dampers for mitigation of such vibrations is used because it could provide mutually interconnected results: reduction of undesired vibration levels and generating of additional electrical power. The examples of their installations into driveline and chassis structures are studied.
\end{abstract}

Keywords: amphibious all-terrain vehicle, vibration, energy harvesting, damper.

\section{Introduction}

The severe vibration fields are a problem for the all-terrain amphibian vehicles during operation in agricultural environment. The purpose of this study is to present the new apparatus for vibration suppression combined with parallel generation of electrical power (energy harvesting) using suppressed vibrations. New midsize all-terrain amphibian vehicle is an object of study. The accumulated experience demonstrates that the harshest vibrations are registered in the driveline and the chassis. The present technology is widely using the energy harvesting units having a certain oscillating weight allowing (in parallel to vibration mitigation) capture, convert and transfer some amount of wasted power [1-5]. Energy harvesting is used in particular for feeding autonomous electronic devices in vehicles. The authors invented and developed Vibration Energy Harvesting Dampers (VEHD) [6;7] consisting of a tuned mass damper (TMD) and alternator or generator of electricity. Detailed analysis of various published materials regarding VEHD is not a subject of the study and the authors decide to concentrate only on own results. The specific of the proposed VEHD is to provide self-tuning to dampen harmonics over a wide interval of fluctuated frequencies using the control system of TMD. The implementation of VEHD in all-terrain amphibian vehicles was dictated by two combined reasons: necessity of reduction of intensive vibrations and proper usage of damped vibration energy while the vehicle engine is operating in a significantly broad interval of changing RPM.

\section{Materials and methods}

A necessity to avoid intensive vibration problems during off-road exploiting of amphibian allterrain vehicles in rural /agricultural areas brings new ideas in schematic composition of such vehicles. The authors developed a new vehicle using some conceptual of the track loader design described in [8] (see Fig. 1).

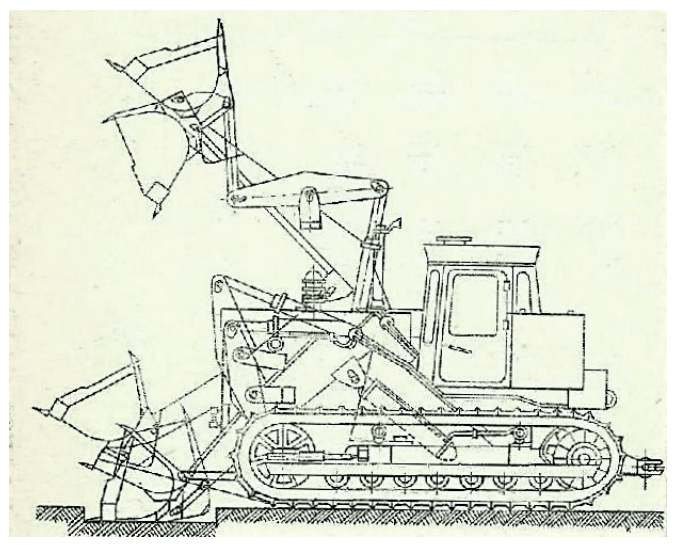

Fig. 1. Track loader with $102.9 \mathrm{~kW}$ engine [8] 
The new vehicle has the same platform concept. However, it is obvious that new parameters were improved and differed from the data issued in [8]. The platform design could be used in several modifications. The authors designed it in form of an amphibian all-terrain vehicle (see photo in Fig. 2).

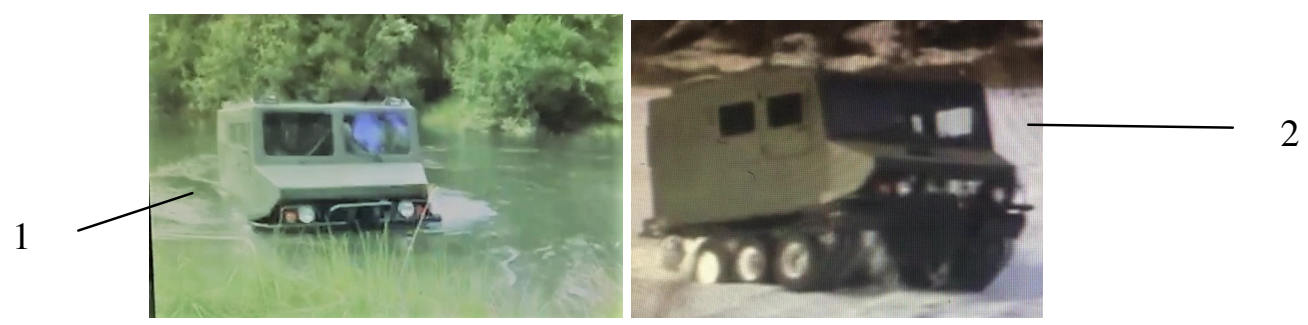

Fig. 2. Amphibious all-terrain track "WOODDZOR": 1 - summertime; 2 - wintertime

Its general specification is - length $3.8 \mathrm{~m}$, width $2.4 \mathrm{~m}$, height $2.15 \mathrm{~m}$; equipped weight $=1850 \mathrm{~kg}$, and total weight $=2600 \mathrm{~kg}$; passenger capacity $=6$ people and $150 \mathrm{~kg}$ of cargo or $750 \mathrm{~kg}$; engine $(1.5-$ litre, Gasoline, 4-cylinder) completed with six-speed automatic transmission; power in range of 120$140 \mathrm{~kW}$; maximum speed on land $=60 \mathrm{~km} \cdot \mathrm{h}^{-1}$; speed on water $=6 \mathrm{~km} \cdot \mathrm{h}^{-1}$; fuel tank $=130$ litres. The vehicle could be equipped by loading/transporting units or other devices. It is an all-season vehicle ready to overcome small river, lake, marsh, snow, and other agricultural circumstances. It was established that the driveline and chassis were the most problematic units of the vehicle from the vibration point of view. Driveline consists technically of two major units: an engine and transmission. The torsional vibrations of the crankshaft (in the engine) and various geared shafts (in transmission) are regular problems for the driveline. The rectilinear vibrations are the problems for the transmission body and the chassis.

The authors proposed the invented universal tool for suppression of different types of vibrations based on VEHD [6;7]. VEHD could be presented in two general versions: "angular - torsional operation" and "rectilinear vibration operation". The detailed scheme descriptions, kinematic and dynamic analysis of VEHD used in the engine are described in [3;7] and [9]. Let us apply now the same approach for suppression of torsional vibrations occurred in transmission.

The general model of transmission is shown in the left part of Fig. 3. The model contains transmission, completed with a flywheel on the entrance end and VEHD on the exit end. The flywheel and VEHD are rotating at the starting point with angular speed $\omega$. Transmission has a system of geared shafts modelled by a coil spring connecting the flywheel and VEHD. It is assumed that the solid part 1 (see the scheme on the right side of Fig.3) of VEHD mounted on the exit shaft. The mass 3 of TMD is attached to the hub of the VEHD solid part 1 by means of a spring 2, so the mass could move in $Z$ direction. Actually, there are several masses located in a symmetrical way for avoiding the dynamic unbalance, and they are composing the torsional vibration damper.

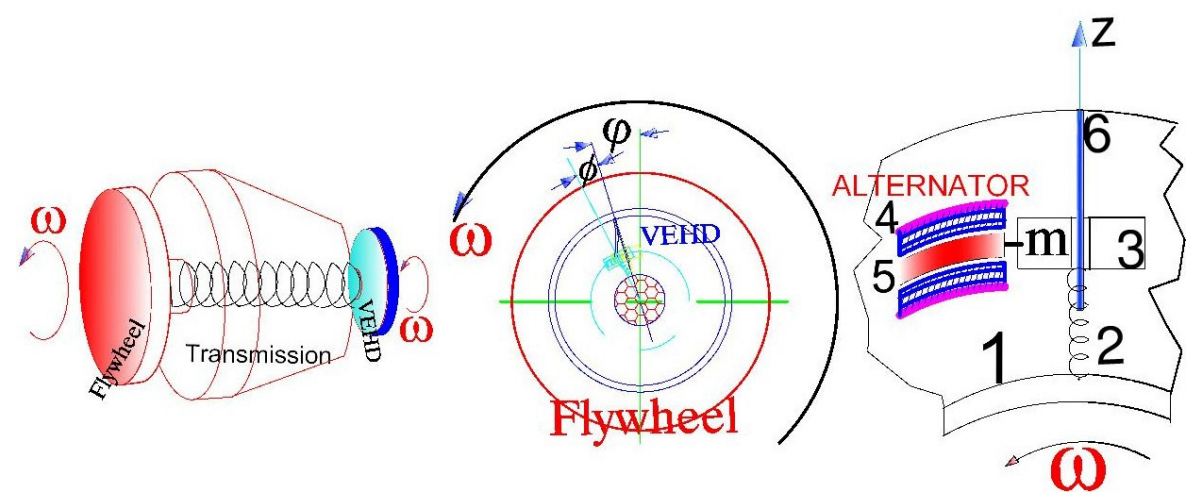

Fig. 3. Modelling of system "flywheel - transmission - VEHD"

Mass 3 is mounted freely on the beam 6 (cantilever spring), which is connected to the outer ring of 1 , but is free at the near hub end of 1 , so between the hub and spring 6 there is a gap. The mass 3 is moving along the beam 6 . Hence, mass 3 could move in radial direction using the beam 6 and oscillating across the clamp point of the beam 6 to the rim. The position of mass 3 is dictated by 
balance of two forces: centrifugal force (trying to move mass 3 to periphery) and the force of the spring 2 compression (trying to keep mass 3 close to the hub). As the radial position of the mass 3 is extended, the length of the cantilever spring 6 for providing the bending beam resistance is effectively shortened. The shortening of the beam, in turn, implies a greater perpendicular force, for perpendicular displacement, i.e. as the length of the bending beam is shortened, the spring constant of proportionality relating displacement to force 6 increases. These effects, the outward displacement of mass 3 under angular velocity and the corresponding increase of the spring constant of proportionality 6 as the masses 3 move outward, result in reducing of oscillations over the operating range of $\omega$.

The solid part of VEHD is vibrating in the same direction as the flywheel, and its displacement coordinate against the flywheel is $\varphi(t)$, where $t$ is the time (see the central part of Fig. 3). Mass 3 is connected to the magnet 5, which is displacing in coils 4 (coils 4 and magnets 5 are the alternator portion of VEHD). The angular coordinate of vibrations of the damper mass 3 with magnet 5 vs. the solid parts of VEHD is $\phi(t)$. The invented device allows to keep automatically the natural frequency $\omega_{\mathrm{o}}$ equal to $\omega^{2}=k_{1} / j=\omega_{\mathrm{o}}^{2}$, where $k_{1}$ is the current stiffness coefficient of the springing elements 6 and $\mathrm{j}$ is the mass moment of inertia of all masses 3 coupled to magnets 5 presented in the VEHD.

Suppose that $\mathrm{J}$ is a mass moment of inertia of all parts located between the flywheel and torsional vibration damper (consisting of masses 3 and magnets 5), which is a mechanical component of VEHD. The stiffness properties of the system of geared shafts modelled by the coil spring are modelled by the stiffness coefficient matrix $k$ and viscous friction matrix coefficient of loss $b$.

The movable parts of VEHD are connected to the solid parts of VEHD by means of springs having the stiffness $k_{1}=j \omega^{2}$ and viscosity factor $b_{1}$. The simplified system of ordinary differential equations for the system-transmission - VEHD" could be written in a similar way to the system presented in [1-4;9;10]. The external force would be presented by $H \sin \omega t$, where $H$ is the magnitude of the external force, $\omega$ is the frequency of the external force. The displacement masses 3 can be ignored for stable regime with constant $\omega$ at considered moment. Regarding the alternator portion of VEHD: its electromagnetic subsystem is inducing current $i$, and $L$ is inductance, $R$ is electrical resistance, where $R_{c}$ is coil resistance and $R_{\mathrm{d}}$ is load resistance, and $R=R_{\mathrm{c}}+R_{\mathrm{d}}$. Hence:

$$
\begin{gathered}
J \ddot{\varphi}+b_{1}(\dot{\varphi}-\dot{\phi})+j \omega^{2}(\varphi-\phi)+b \dot{\varphi}+k \varphi=H \sin \omega t \\
j \ddot{\phi}-b_{1}(\dot{\varphi}-\dot{\phi})-j \omega^{2}(\varphi-\phi)+F i=0, \\
L \dot{i}+R i-F(\dot{\phi}-\dot{\varphi})=0,
\end{gathered}
$$

where $\dot{\varphi}=d \varphi / d t$, and $F$ - linkage factor.

The linkage factor could be determined in most cases as $F=B L_{\mathrm{s}}$, where $B$ is the field flux and $L_{\mathrm{s}}$ is the characteristic size. The structure of equation (1) in the system (1) - (3) reflects the fact that transmission is considered like a one-degree-of-freedom subsystem. It should be changed, when the examined subsystem could be identified as a multi-degree-of-freedom subsystem, for instance, twodegree-of-freedom subsystem; in the case of two-degree-of-freedom the equation (1) would be split in two equations.

Before starting the numerical simulation analysis the peak-to-peak twist angles of the real sixspeed automatic transmission were recorded for running the vehicle at $50 \mathrm{~km} \cdot \mathrm{h}^{-1}$.

The preliminary recorded peak-to-peak twist angles of transmission are presented in Figure 4A, where the abscissa axis is the frequencies in $\mathrm{Hz}$, and the ordinate axis the $2 \varphi$ torsional peak-to-peak exit end angle vibration values (in degrees) are shown. The range of interest $20-70 \mathrm{~Hz}$ was selected taking into an account the results of preliminary estimates of transmission low mode resonance frequencies by FEM.

The analysis of the obtained data shows that there are several spikes of twist angles; however, the biggest values of the recorded peak-to-peak twist angle are 1.256 degrees at $52.5 \mathrm{~Hz}$ and 0.623 degrees at $63 \mathrm{~Hz}$. The recorded values are higher than the required 0.4 degrees [9]. Hence, it could be suggested that for modelling purposes it would be possible to use a two degree-of-freedom model for considering torsional vibrations in transmission. 

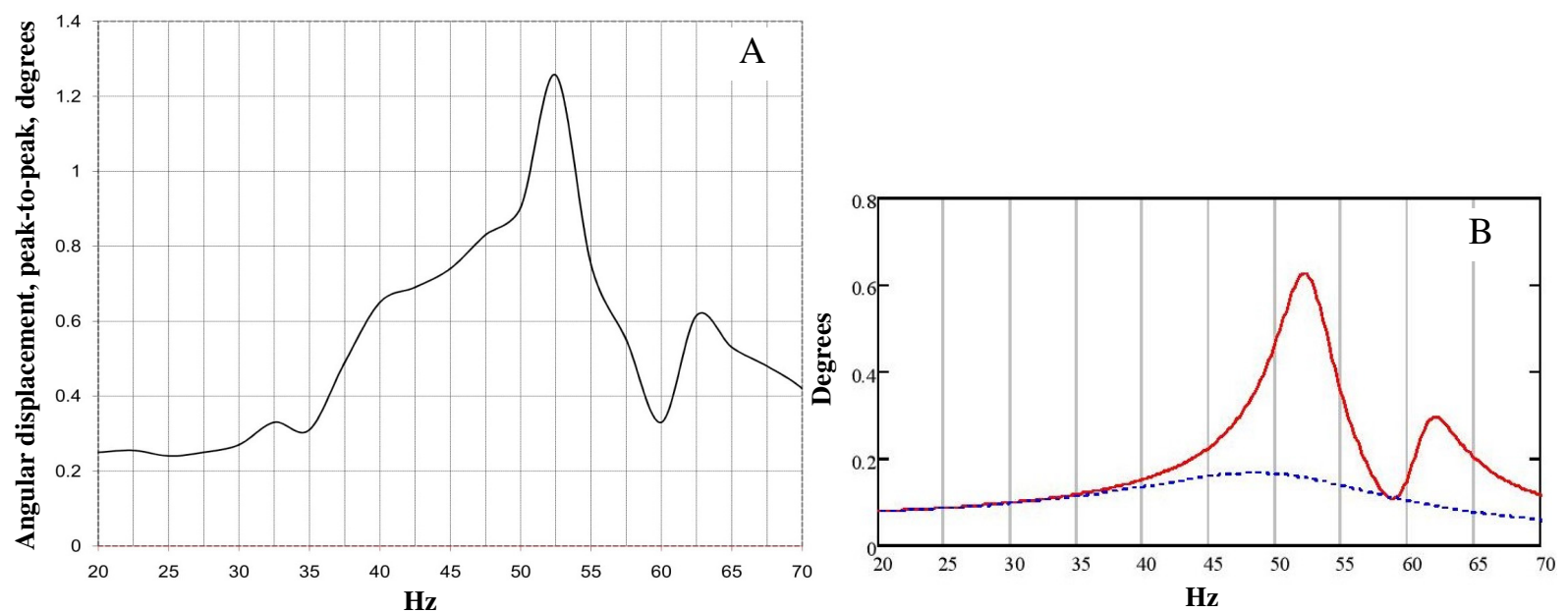

Fig. 4. A - recorded Peak-to-Peak Twist Angles of tested transmission; B - angular amplitudefrequency characteristics for torsional vibrations of transmission

\section{Results and discussion}

Now the transmission torsional vibration model is presented by two a degree-of-freedom subsystem, it means that equation (1) is split into two interconnected equations (1A) and (1B); the torsional vibration damper component and alternator would be presented formally by equations (2) and (3). The selection of the model parameters is dictated by the following circumstances: the shape of the angular amplitude-frequency characteristic must be close to the shape of the recorded graph of the peak-to-peak twist angles (Fig. 4A), and the maximal recorded peak in Figure 4A must coincide with the modelled peak. Using these criteria, it is possible to build the angular $(\varphi)$ amplitude-frequency characteristic as shown in Figure $4 \mathrm{~B}$ by the red solid curve. The abscissa horizontal axis is for excitation frequency in $\mathrm{Hz}$ and the ordinate vertical axis in Figure $4 \mathrm{~B}$ is for angle amplitude in degrees. Actually, the red curve reflects the original system dynamical behaviour (no VEHD installed). Analyzing the amplitude-frequency characteristic for the system in Figure 4B, it is obtained that the peak amplitude of 0.628 degrees is presented at excitation frequencies equal to $52.5 \mathrm{~Hz}$; it is equivalent to the spike of $2 \varphi$ in Figure 4A. The second spike of 0.308 degrees is located at $62 \mathrm{~Hz}$. One can state that the selected model of the transmission torsional vibrations is equivalent to a real low frequencies mechanism.

The obtained data were used for designing of VEHD applicable for that specific transmission. Also, it was assumed that the mass moment of inertia $j$ of the movable parts of VEHD $j=0.018 \mathrm{~J}$. Let us suppose for simplicity that the initial parameters of an alternator for trial study are selected following the recommendations [3;4]. The theoretical angular amplitude - frequency characteristic of transmission equipped by such VEHD is shown in Figure 4B by the blue dotted curve. The peak of the damped torsional vibration curve is equal to 0.168 degrees at $49 \mathrm{~Hz}$. No other peaks are for this curve. Now the maximal peak-to-peak twist angle $(2 \varphi)$ is equal to 0.336 degrees. So, for total range of operating RPM (frequencies) the torsional vibrations of the engine shaft completed by VEHD are lower than the torsional vibrations of the engine shaft without VEHD.

Thinking about reduction of the torsional vibration level at initial resonance $(52.5 \mathrm{~Hz})$, one can see that the amplitude of the system with VEHD at $52.5 \mathrm{~Hz}$ is equal to 0.157 degrees now, so the rate of amplitude reduction at the initial system resonance (@52.5 Hz) is 4 times. VEHD maximum induced power would be equal to $62.21 \mathrm{~W}$.

The prototype of the proposed transmission VEHD was designed, fabricated, assembled and tuned (see photo of the prototype presented in Fig. 5A). The recorded peak-to-peak twist angles of transmission with VEHD are presented in Figure 5B, where the abscissa and ordinate axis are similar to the plot in Fig 4A. The analysis of these graphs shows that there is one peak of twist angles -0.367 degrees at $48 \mathrm{~Hz}$. The maximum recorded induced electrical power is $59 \mathrm{~W}$. The obtained results prove the accuracy of the selected modelling. 

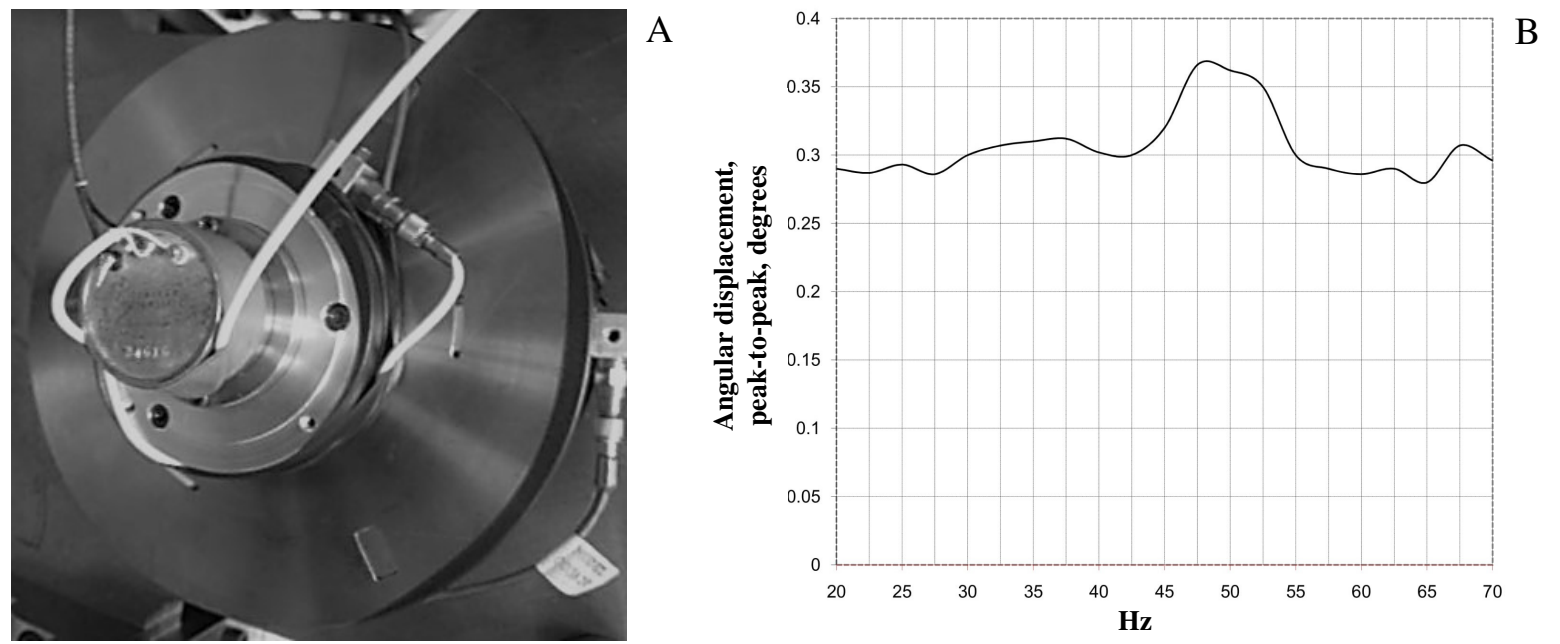

Fig. 5. A - transmission VEHD; B - recorded Peak-to-Peak

Twist Angles of transmission with VEHD

A similar approach was applied for suppression of the engine crankshaft torsional vibrations. The prototype incorporating the features and properties of the angular torsion version of VEHD was developed, manufactured and installed in the front end of the engine shaft. The operation range of rpm for the engine was 800-5,000. The concerned resonance was recorded on a graph for $6^{\text {th }}$ order torsional vibrations at $4100 \mathrm{rpm}$ with the peak-to-peak angle of 1.002 degrees. It means that actual crankshaft torsional vibrations with 1.002 degrees (despite of the presence of regular Torsional Vibration Damper) were at $\omega=4100(\mathrm{rpm}) \times 6$ (order) $\times \pi / 30=2574.81 / \mathrm{sec}=410 \mathrm{~Hz}$. The installation of VEHD leads to the following results: there are $6^{\text {th }}$ order resonance torsional vibrations @ 3550 RPM with the peak-to-peak angle of 0.127 degrees. It is significantly lower than the industrial requirement of 0.4 degrees. The maximal value of the recorded generated power was very small $-1.1 \times 10^{-3} \mathrm{~W}$.

For mitigation of undesired chassis vertical vibrations the rectilinear version of VEHD was designed. The differences between the "angular - torsional operation" version of VEHD and "rectilinear vibration operation" version are: 1) formally the rectilinear model has masses M and $\mathrm{m}$ instead of the masses moment of inertia $J$ and $\mathrm{j}$ in equations $(1)-(3) ; 2)$ the rectilinear version must be equipped by an additional control system for keeping $\omega^{2}=k_{1} / m=\omega_{0}^{2}$.

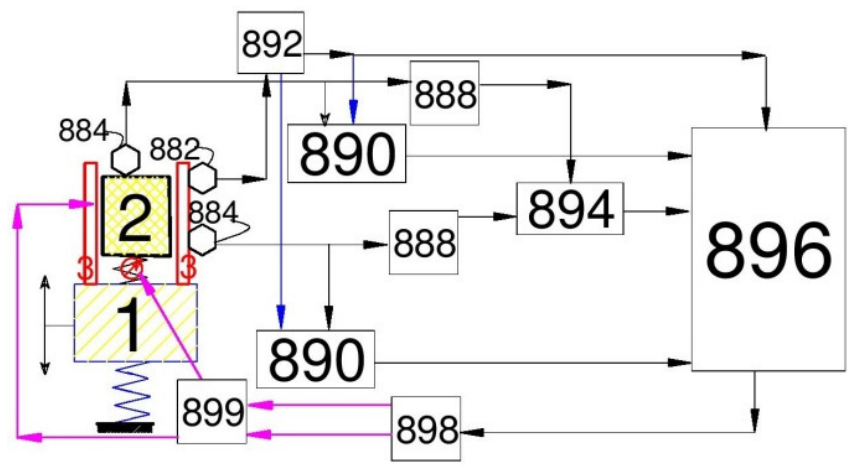

Fig. 6. Schematic diagram of electromagnetic apparatus version for rectilinear VEHD

Fig. 6 shows one of the schematic diagrams of an electromagnetic apparatus $\mathbf{8 0 0}$ for providing feedback to the dynamic system 1-2-3 for keeping $\omega^{2}=k_{1} / m=\omega_{0}{ }^{2}$. Accelerometers 884 are connected to 2 - TMD and to 3 - electromagnetic solenoid (shown in cross section). The accelerometers $\mathbf{8 8 4}$ are each connected to integrators $\mathbf{8 9 0}$. Both outputs of the integrator $\mathbf{8 9 0}$ are connected to the computing system 896. The accelerometers $\mathbf{8 8 4}$ are also connected to the phase detectors $\mathbf{8 8 8}$ that are, in turn, connected to the one phase discriminator $\mathbf{8 9 4}$. This is, in turn, connected to the computing system $\mathbf{8 9 6}$. Also connected to the solenoid is the frequency detector $\mathbf{8 8 2}$ that is connected, via filter $\mathbf{8 9 2}$, to the integrators 890 and the computing system 896. Amplifiers and filters may be used where needed for ensuring proper signal transmission. The computing system is connected to the signal generator $\mathbf{8 9 8}$, 
which is connected to the actuator of the springing element (located between 1 and 2) and solenoid $\mathbf{3}$. The computing system $\mathbf{8 9 6}$ has the capacity to (i) input frequency, amplitude and phase difference data, (ii) perform algorithms on this data, and (iii) generate command signals to govern VEHD. The computing system 896 accommodates the designed algorithm, which provides self-tuning.

The prototype of VEHD focused on extracting the possibly maximum power from vibrating chassis was designed and fabricated. The design parameters were composed specifically for prototype mounting on a chassis vibrating in the range of $16.5-25.0 \mathrm{~Hz}$. The selected mass of the fabricated TMD part in VEHD was $1 / 6$ of model mass or $1.05 \mathrm{~kg}$. All other main characteristics were based on values relatively presented in the considered modelling case. Six units of VEHD were vertically installed close to the perimeter of the chassis. Comparison of the values recorded in the tests with the data obtained in the previous theoretical study was done. It is necessary to underline at this moment that it would be expected that six properly located equal-size distributed VEHD of fabricated mass of $1.05 \mathrm{~kg}$ should substitute one VEHD having the mass of $6.3 \mathrm{~kg}$. The measured maximum amplitude of the chassis vibrations before installation of VEHD was registered at $3.8 \mathrm{~mm}$. The measured maximum amplitude of the chassis vibrations with assembled VEHD was $2.4 \mathrm{~mm}$. The recorded average magnitudes of useful powers of each of six VEHD were summarised and the result was $2.47 \mathrm{~kW}$. It is around $2 \%$ of the working engine power. It could be underlined that the presented configuration of VEHD is not restricted, and VEHD could be applicable in various situations in all types of vehicles. All types of vehicles have more or less similar problems with the chassis because of intensive agricultural loads. Hence, all chassis need to be equipped by the newly designed VEHD.

\section{Conclusions}

1. Effective protection of all-terrain amphibian vehicle major components such as the engine, transmission and chassis from severe vibration fields combined with obtaining additional electrical power would be reached by implementation of the patented VEHD. The elaborated VEHD has several improved features being installed on the selected unit of all-terrain amphibian vehicles exploited in agriculture. The universality of application of the proposed schemes is one of the most important issues among others.

2. There could be a recommendation regarding the usage of VEHD specifically in the agricultural amphibian all-terrain vehicles - implement VEHD focused on the effectiveness of using wasted power for obtaining additional portions of electrical power.

3. The developed VEHD should be installed and used successfully in various types of vehicles. The proposed VEHD could be used for feeding autonomous electronic devices and/or charging onboard batteries.

\section{Acknowledgements}

This work was supported by the Russian Science Foundation under the grant no 19-19-00065.

\section{References}

[1] Priya S., Inman D. L. Energy Harvesting Technologies. Sprinder. Verlag, 2009.

[2] Cottone F. Energy Harvesting: introduction in NiPS Summer School, Fiuggi (Italy), 2015, 50 p.

[3] Bograd A., Nerubenko G., Nerubenko C. Energy Harvesting in Vehicle's Drive. Proceedings of the 2nd International Conference of Energy Harvesting, Storage, and Transfer (EHST'18). Niagara Falls, Canada, Paper No. 112, 2018.

[4] Nerubenko G., Bograd A., Kolomier P. Tuned mass dampers as energy harvesters for railways. Proceedings of ISMA2018 and USD2018. Leuven, Belgium, 2018 pp. 1689-1702.

[5] Cottone F., Goyal S., Punch J. Energy harvester apparatus having improved efficiency. US Patent 8,350,394. January 8, 2013.

[6] Nerubenko et al. Vibration Energy Harvesting Damper. U.S. Patent Application No. 16/119, 346, 2018.

[7] Nerubenko G. Torsional Vibration Damper of a Rotating Shaft. US Patent 7,464,800. Dec.. 16, 2008 . 
[8] Нерубенко Г. П., Ватипко Б. А., Щур П. А., Справочник по перегрузочному оборудованию на морском транспорте (The handbook of loading equipment in marine transport), Одесса Маяк 1990, 221 p. (In Russian).

[9] Nerubenko G. Tuned Torsional Vibration Damper. SAE Technical Paper 2013-01-1897, 2013, doi: 10.4271/2013-01-1897.

[10] Nerubenko G., Krupenin V., Nerubenko C. Vehicle Hybrid Free - Piston Engine - Generator. 16th International Scientific Conference "Engineering for Rural Development". Proceedings. Jelgava, Latvia, May 24.-26, 2017. pp. 114-120. 